\title{
EDITORIAL
}

\section{Sanofi-Cell Research outstanding paper award of 2018}

\author{
Cell Research (2019) 29:873-874; https://doi.org/10.1038/s41422-019-0248-2
}

We are pleased to announce winners of the 2018 Sanofi-Cell Research Outstanding Research Article Award: Drs Pan Zheng and Yang Liu, for their back-to-back papers entitled "A reappraisal of CTLA-4 checkpoint blockade in cancer immunotherapy" and "Uncoupling therapeutic from immunotherapy-related adverse effects for safer and effective anti-CTLA-4 antibodies in CTLA4 humanized mice"; Dr Xiao-Fan Wang, for his paper entitled "Switching off IMMP2L signaling drives senescence via simultaneous metabolic alteration and blockage of cell death"; and Drs Jinsong Li, Fuchou Tang and Ming-Han Tong, for their paper entitled "Single-cell RNA-seq uncovers dynamic processes and critical regulators in mouse spermatogenesis". Each award consists of a prize of $€ 5000$ sponsored by Sanofi.

The classic checkpoint blockade hypothesis states that cancer immunity is restrained by two distinct checkpoints: the CTLA-4:B7 interaction and the PD-1/B7-H1(PD-L1) interaction. It is assumed that anti-CTLA-4 antibodies cause tumor rejection by blocking negative signaling from B7-CTLA-4 interactions. In the first award-winning research article, published in the April 2018 issue, Drs Zheng, Liu and colleagues $^{1}$ showed that the mechanisms of therapeutic anti-CTLA-4 antibodies are independent of checkpoint blockade but dependent on Treg depletion and the host Fc receptor. In their second paper, ${ }^{2}$ they demonstrated that complete CTLA-4 occupation, systemic T cell activation and preferential expansion of self-reactive $\mathrm{T}$ cells are dispensable for tumor rejection but correlate with immunotherapyrelated adverse events. Taken together, these two papers provided important insights for clinical development of safer and potentially more effective CTLA-4-targeting immunotherapy. Cellular senescence is a fundamental cell fate playing an important role during the natural aging process. In the second award-winning research article, published in the June 2018 issue, Dr Wang and colleagues ${ }^{3}$ showed IMMP2L-mediated signaling pathway drives the senescent process by reprogramming mitochondria-associated redox status, phospholipid metabolism and signaling network, and simultaneously blocking cell death under oxidative stress conditions. This study uncovered that loss of IMMP2L signaling represents one of the key events associated with cellular senescence as alteration of this network can be observed across a variety of tissue and cell types. Mammalian spermatogenesis is a complex, asynchronous process which involved dramatic morphological and biochemical changes. In the third award-winning research article, published in the September 2018 issue, Drs Li, Tang, Tong and colleagues ${ }^{4}$ defined molecular events across male germ cell development by using single-cell RNAseq to analyze individual synchronous and homogenous spermatogenic cells. This paper provides a valuable resource for future studies towards the complete elucidation of gametogenesis.

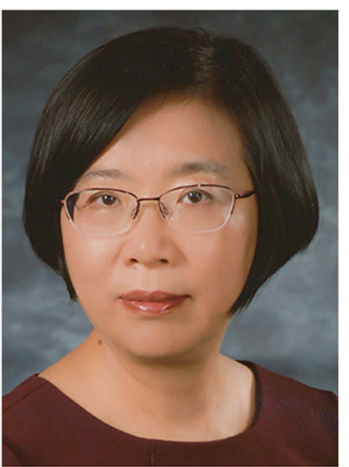

Dr Pan Zheng

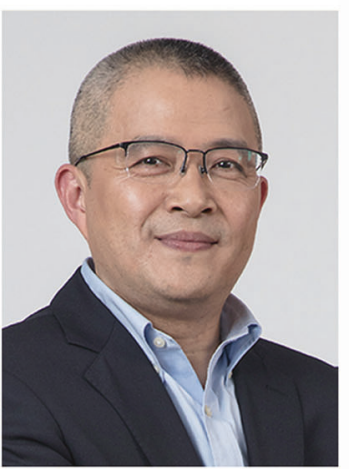

Dr Jinsong Li

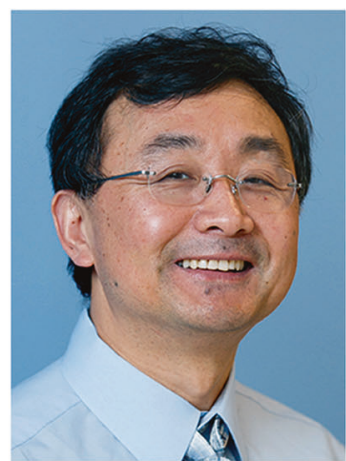

Dr Yang Liu

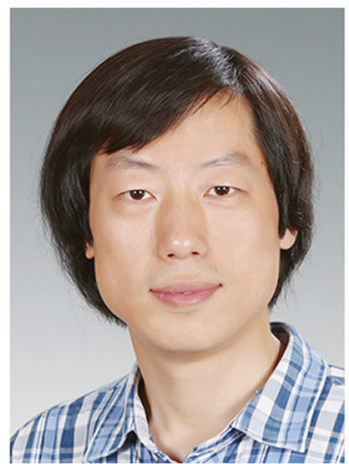

Dr Fuchou Tang

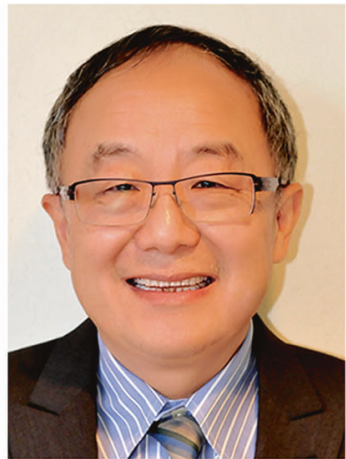

Dr Xiao-Fan Wang

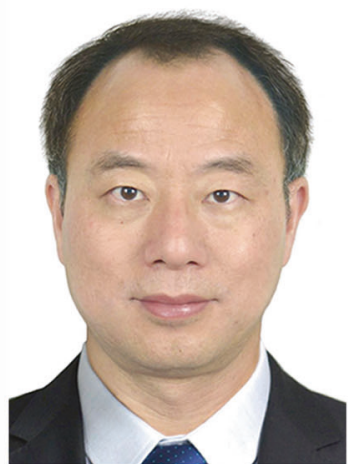

Dr Ming-Han Tong 
Please join us to congratulate Drs Zheng and Liu, Dr Wang, and Drs Li, Tang and Tong on their winning of the 2018 Sanofi-Cell Research Outstanding Paper Award.

Cell Research Editorial Team ${ }^{1}$ ${ }^{1}$ Shanghai Institute of Biochemistry and Cell Biology/CAS Center for Excellence in Molecular Cell Science, Chinese Academy of Sciences, Shanghai 200031, China Correspondence: cellres@sibcb.ac.cn

\section{REFERENCES}

1. Du, X. et al. A reappraisal of CTLA-4 checkpoint blockade in cancer immunotherapy. Cell Res. 28, 416-432 (2018).

2. Du, X. et al. Uncoupling therapeutic from immunotherapy-related adverse effects for safer and effective anti-CTLA-4 antibodies in CTLA4 humanized mice. Cell Res. 28, 433-447 (2018)

3. Yuan, L. et al. Switching off IMMP2L signaling drives senescence via simultaneous metabolic alteration and blockage of cell death. Cell Res. 28, 625-643 (2018).

4. Chen, Y. et al. Single-cell RNA-seq uncovers dynamic processes and critical regulators in mouse spermatogenesis. Cell Res. 28, 879-896 (2018). 